DOI: $10.33947 / 1980-6469-v 15 n 1-4003$

\title{
AS CONSEQUÊNCIAS DO BULLYING: AUTOAGRESSÃO E SUICÍDIO NO COTIDIANO ESCOLAR
}

\section{THE CONSEQUENCES OF BULLYING: SELF-AGGRESSION AND SUICIDE IN SCHOOL LIFE}

\author{
Elizete Prescinotti Andrade ${ }^{1}$, Lilia D’souza Li²
}

\section{RESUMO}

A proposta dessa revisão foi analisar a relação do bullying com a autoagressão não suicida e o suicídio e verificar quais os possíveis mecanismos que ligam o bullying a essas duas situações. A prevalência do bullying nas escolas vem aumentando, inclusive no Brasil, e diversos estudos mostram que estar envolvido em atos de bullying, principalmente como alvo, é fator de risco para desenvolver estresse tóxico. Esse estresse leva a liberação glicocorticoide em concentrações maiores que a fisiológica e por vezes por períodos prolongados. Essa desregulação na quantidade de glicocorticoide tem a capacidade de mudar a cito arquitetura cerebral. O glicocorticoide age em regiões do cérebro ligada à memória, ansiedade, tomada de decisões e a cognição. Os estudos mostram que estar envolvido em atos de bullying está significativamente relacionado a depressão, autoagressão não suicida e com suicido. Bullying é um tipo violência, e um importante problema de saúde pública que exige a atenção coordenada de profissionais de saúde, educadores, famílias e gestores públicos.

PALAVRAS-CHAVES: Bullying. Suicídio. Depressão

\section{ABSTRACT}

The purpose of this review was to analyze the relationship of bullying with non-suicidal self-harm and suicide and to verify the possible mechanisms that link bullying to these two situations. The prevalence of bullying in schools has been increasing, including in Brazil, and several studies show that being involved in acts of bullying, especially as a target, is a risk factor for developing toxic stress. This stress leads to glucocorticoid release at concentrations greater than physiological and sometimes for prolonged periods. This deregulation for glucocorticoid has the ability to change the cerebral architecture. Glucocorticoid acts in regions of the brain linked to memory, anxiety, decision-making, and cognition. Studies show that being involved in bullying is significantly related to depression, non-suicidal selfharm and suicide. Bullying is a type of violence, and an important public health problem that requires the coordinated attention of health professionals, educators, families and public managers.

KEYWORDS: Bullying. Suicide. Depression

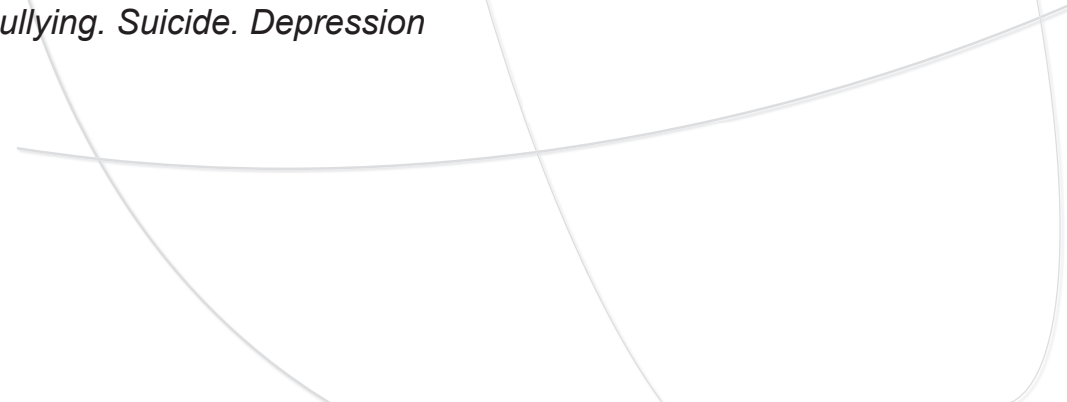

1 Médica Pediatra. Especialista em Pediatria pela Sociedade Brasileira de Pediatria e Associação Médica Brasileira. Habilitação em Medicina do Adolescente pela Sociedade Brasileira de Pediatria e Associação Médica Brasileira. Mestre em Ciências (Área de Concentração: Pediatria) pela Universidade Estadual de Campinas. Colaboradora do Ambulatório de Medicina do Adolescente, do Departamento de Pediatria da Universidade Estadual de Campinas. Autora correspondente: elizete. prescinotti@uol.com.br

2 Professora Lília de Souza Li, Doutora em Medicina Experimental pela McGill University, Canadá, professora assistente doutora do Departamento de Pediatria, professora plena do Programa de Pós-Graduação em Saúde da Criança e do Adolescente, pesquisadora do Centro para Investigação em Pediatria (CIPED), Faculdades de Ciências Médicas, Universidade de Campinas, UNICAMP. 


\section{Introdução}

Bullying é uma forma multifacetada de violência, que se tornou um importante problema de saúde pública e exige a atenção coordenada de profissionais de saúde, educadores, famílias e gestores públicos. Atualmente, existe um maior conhecimento sobre a morbidade e mortalidade associadas ao bullying, mas ainda são tímidas as ações para interromper essa cadeia de violência que a longo prazo, pode interferir com a saúde mental de toda uma geração de indivíduos que ingressarão no mercado de trabalho e com isso alterar o progresso de uma nação.

Embora o problema de bullying entre estudantes ocorra muito provavelmente há séculos, foi somente no início da década de 1970 que Dan Olweus iniciou a primeira pesquisa sistemática sobre este problema. Os resultados foram publicados em um livro na Suécia em 1973 e nos Estados Unidos em 1979 sob o título "Agressão nas Escolas: intimidações e agressões em meninos" (OLWEUS,1979). Por um período de tempo considerável, até o início de 1990, houve pouca atenção e pesquisa sobre o tema fora da Escandinávia. Em 1983, depois que três meninos adolescentes no norte da Noruega suicidarem-se, muito provavelmente como consequência da grave intimidação por parte de colegas, o Ministério da Educação daquele país iniciou uma campanha nacional contra o bullying nas escolas. Neste contexto, a primeira versão do que mais tarde se tornou conhecida como o Programa Olweus de Prevenção de Bullying foi desenvolvido (OLWEUS,1993).

D. Olweus sempre entendeu o bullying como uma violação aos direitos humano e em 1981 ele propôs a promulgação de uma lei contra o bullying nas escolas. Ele argumentou que é um direito fundamental do estudante sentir-se seguro na escola e ser poupado da repetida humilhação que está implícita no bullying. Em meados da década de 1990, estes argumentos levaram à criação de uma legislação contra o bullying pelos parlamentos da Suécia e Noruega. Legislação semelhante foi adotada por mais de 28 estados nos Estados Unidos, e em vários outros países (CORNELL E LIMBER, 2015).

Existe grande variabilidade na prevalência de bullying nos diversos países em decorrência de usos de metodologias diferentes, mas se tomarmos o Develo- ping a Global Indicator on Bullying of School-aged Children, UNICEF 2013 onde 150 milhões de adolescentes com idade entre 13 e 15 anos referiram terem sido alvos de bullying na escola ou ao redor dela, percebemos que o bullying é muito prevalente (Unicef, 2013). Outro estudo em parceria da Unicef com OCDE intitulado "Um começo desleal: desigualdade na educação de crianças em países ricos" encontrou que a taxa de bullying, variou de $25 \%$ na Finlândia a $60 \%$ na Nova Zelândia; nos Estados Unidos da América essa taxa foi de $45 \%$. Um em cada três (33\%) alunos disseram que foram vítimas de bullying semanalmente, evidenciando que o bullying é um problema difícil de resolver, mesmo em países que têm melhores índices referentes às escolas e ao aprendizado (UNICEF,2018).

No Brasil após a instituição da Pesquisa Nacional de Saúde Escolar realizadas nos anos de 2009, 2012 e 2015, temos como acompanhar a evolução da prevalência, pois utiliza a mesma metodologia. E nesse estudo encontramos a seguinte taxa de bullying, $14,2 \%$ (2009), 16,5\% (2012) e 21,7\% (2015) (AZEREDO et al, 2019).

Com esses aumentos evidentes da taxa de bullying e as evidencias encontradas em vários estudos entre bullying e depressão (KODISH et al 2016), bullying e suicídio (KLOMEK et al, 2013) faz necessários que todos os profissionais que trabalhem com crianças e adolescentes aprofundem o conhecimento na correlação entre o bullying e o sofrimento psíquico. Alvos de bullying tem chances significativamente maiores de desenvolver problemas de saúde mental em comparação com não alvos bullying (Benedict, Vivier e Gjelsvik, 2015). Da mesma forma crianças e adolescentes com sintomas depressivos e ou de ansiedade tem chance significativamente maior de serem alvos de bullying (Albuquerque, Williams e D' Affonseca e 2013).

\section{Estresses tóxico associado ao bullying}

Há evidências crescentes que estar envolvido em atos de bullying seja como alvo, agente ou testemunha está associado ao estresse tóxico, e pode levar a consequências psicológicas adversas de longa duração (MELLO et al, 2017).

Essa associação é muito mais evidente quando se refere aos alvos de bullying, levando a resultados 
acadêmicos ruins, prejuízo das relações sociais, baixa autoestima, problemas na cognição e a comprometimento da saúde física e psíquica (BELLMORE E CILLESSEN, 2006; ESBENSEN E CARSON, 2009; Naveed et al,2019).

Um certo grau de estresse é necessário para que os indivíduos se adaptem ás mudanças ambientais e respondam adequadamente a ameaças ou perigos imediatos. A eficiência dessa resposta está ligada a capacidade de memorizar e correlacionar informações aprendidas anteriormente, a fim de responder efetivamente a demandas similares no futuro. Ou seja, o estresse tem que ser suficiente para levar a formação de memória de longa duração (JUVONEN, WANG E ESPINOZA, 2011; FINSTERWALD E ALBERTINI, 2014; DU PLESSIS et al, 2019).

Nós seres humanos estamos sujeitos a três tipos de estresses, este descrito anteriormente, que é essencial para nosso desenvolvimento, nominado como estresse leve. Um segundo tipo, estresse moderado, por exemplo como a perda de um familiar, que se adequadamente amparado pode ocasionar formas benéficas de aprendizagem que promoveram resiliência futura.

O terceiro tipo, o estresse tóxico, que se caracteriza por ser de longa duração, frequente ou de forte intensidade, não responde as atitudes de reforço habituais e tem potencialidade para causar alterações permanentes que podem levar a mudanças comportamentais, cognitivas, fisiológicas e neurais que levam a comprometimento da saúde física e psíquica (FINSTERWALD E ALBERTINI, 2014).

A infância e adolescência se caracterizam por ser um período em que os indivíduos estão em desenvolvimento, principalmente o cérebro, que em decorrência disso torna-se particularmente sensível às influências químicas. Existem evidências crescentes apoiadas por estudos em animais e humanos, principalmente com neuroimagem, que mostram que a elevação de hormônios do estresse, principalmente os glicocorticoides, podem alterar a arquitetura cerebral (BUCCIM et al, 2016). Por exemplo, receptores de glicocorticoides são encontrados em grande número na amígdala, hipocampo e o córtex pré-frontal. A exposição a experiências estressantes por meio da elevação dos níveis séricos de glicocorticoides alteram o tama- nho e a arquitetura neuronal dessas áreas, interferem na aprendizagem, memória e na adequada execução de tarefas. Mais especificamente, o estresse crônico está associado à hipertrofia e hiperatividade na amígdala e no córtex orbito frontal e perda de neurônios e conexões neurais no hipocampo e no córtex préfrontal medial. As consequências funcionais dessas mudanças estruturais incluem aumento da ansiedade relacionada à hiperativação da amígdala, menor capacidade de tomar decisões de forma adequada, levando a maior risco de assumir comportamentos deletérios a si mesmo ou a outrem, como resultado de atrofia do córtex pré-frontal medial.

A memória de curta e longa duração e o humor ficam prejudicados em função da redução do hipocampo (MC EWEN e GIANAROS, 2011). A amígdala, além de seu papel na mediação do medo e da ansiedade também é um ativador da resposta fisiológica ao estresse estabelecendo um ciclo de feedback positivo, que frente a um estresse, mesmo que não de forte intensidade, leva a uma liberação aumentada de glicocorticoides, mantendo as crianças e adolescentes em um estado de estresse crônico. Estar exposto ao estresse crônico e a altos níveis de glicocorticoides inibe a neurogênese no hipocampo, que além da codificação da memória possuem outras funções (SHONKO$F F, 2012)$. Uma delas que fica muito prejudicada é a capacidade de o hipocampo promover aprendizagem contextual, tornando mais difícil discriminar condições para as quais pode haver perigo versus segurança, como é comum no transtorno de estresse pós-traumático. Esses achados são fortemente correlacionados com o bullying e mostram porque estar envolvido em atos de bullying pode levar a maior risco de desenvolver problemas relacionados à saúde mental e aprendizagem (RIVAEt al,2015; RUDOLPH et al,2016; WILL et al, 2016; TELZER, MIERNICKI e RUDOLPH,2018)

Tem sido proposto que a adolescência é o período do desenvolvimento em que os efeitos de exposições anteriores ao estresse, isto é, infância e período intrauterino, se tornam mais evidentes. Ou seja, estudantes que ao entrarem na adolescência, o fizerem em um estado de estresse crônico, frente a novos estresses responderão com níveis mais elevados de glicocorticoide (LUPIEN et al,2009). Há algumas evidências que sugerem que os níveis de glicocorticoides podem 
ter papel importante no quanto o ato de bullying afeta os envolvidos, por exemplo nos adolescentes alvos de bullying e que apresentavam sintomas depressivos os níveis basais de glicocorticoide eram maiores (CARRION et al, 2010) (RUDOLPH, TROOP-GORDON e GRANGER, 2011) (BRENDGEN et al, 2017). É evidente que existem muitos outros fatores envolvidos na resiliência das pessoas, mas a prevenção do estresse tóxico desde o período intrauterino deve ser uma busca de todos os profissionais envolvidos em cuidar de crianças e adolescentes. As ações protetivas não devem focar apenas na saúde física, mas também na psíquica.

\section{Autoagressão não suicida}

Se refere a uma variedade de comportamentos em que um indivíduo propositalmente inflige danos ao seu corpo sem a intenção de cometer suicídio (HAWTON e HARRIS,2007) (BAKKENE D Gunter,2012). A autoagressão, mais frequente são os cortes intencionais da pele e do tecido subdérmico, mas podem ser também arranhões, queimaduras, ou retirada de pedaços de pele, de cabelo, de unha, ou ainda ingestão de substâncias tóxicas, medicamentos não prescritos ou em doses maiores, hematomas e fratura óssea (MC DOUGALL e VAILLANCOURT, 2015). Embora não seja tipicamente um gesto suicida, a autoagressão está estatisticamente associada ao suicídio e pode resultar em graves danos à saúde física (HAWTON e HARRIS,2007). As estatísticas sobre a autoagressão são ainda díspares, um grande estudo de coorte populacional na Austrália, verificou que $8 \%$ dos adolescentes com menos de 20 anos relataram que se machucaram em algum momento de suas vidas (Moran, COFFEY e ROMANIUK, 2012) É importante relatar que a maioria dos estudos são feitos nas emergências de hospitais e, portanto, nesses estudos estão contabilizados apenas os casos graves de autoagressão. Acredita-se que menos de um quarto das crianças e adolescentes que praticam autoagressão não suicida são diagnosticadas pelos serviços de saúde, a maioria passa desapercebida pelos profissionais de saúde (MARS et al, 2014).

A autoagressão não suicida está estritamente correlacionada com a presença de transtornos de ansiedade e depressão (e com a possibilidade de trans- tornos mentais na vida YSTGAARD et al, 2008). Pesquisa realizada em unidades primárias de saúde que avaliou dados de 16.920 adolescentes de 10-19 anos encontrou um aumento de $68 \%$ na incidência de autoagressão não suicida entre meninas de 13-16 anos entre os anos 2011-2014. Mostrando uma incidência maior no início da adolescência (MORGAN et al,2017). Outra meta analise também encontrou esse mesmo dado, adolescentes mais jovens relataram significativamente mais autoagressão não-suicidas do que adolescentes mais velhos (BERNNARDI et al, 2016).

'A relação entre o bullying e a autoagressão não suicida tem sido bem estabelecida (BERNNARDI et al, 2016), assim como a associação entre autoagressão não suicida, bullying e sintomas depressivos (CLAES et al, 2015) (GARISCH e WILSON, 2015) (BRUNSTEIN et al, 2016). E também tem forte relação com transtornos alimentares (VARELA-BESTEIRO et al, 2017) (SMITHUIS et al, 2018).

É importante salientar que o que leva às autoagressões não suicidas na maioria das vezes, não é um único fator, mas sim a conjunção de vários. Sendo os mais relevantes a violência física, sexual, o abandono em todas suas formas, famílias disfuncionais, doenças psiquiátricas, uso de substâncias psicoativas etc. (HAWTON e HARRIS, 2007).

Os atos de autoagressão em $80 \%$ dos casos ocorrem em casa, no quarto ou banheiro. Na maioria das vezes a família não sabe sobre esses atos, mas os amigos sabem e quando ele se dá por meio de cortes, eles muitas vezes se cortam também. O antebraço é superfície corporal mais usada em cerca de $70 \%$ dos casos. Mas os cortes podem aparecer em outras áreas como abdome, região cervical e áreas mais escondidas. Na tentativa de esconder os cortes a maioria dos adolescentes começam a usar roupas de mangas longas ou evitam se exporem em situações que possam mostrá-los, usam roupas de mangas longas em dias de calor, evitam usar shorts.

O principal motivo alegado para a realização da autoagressão é para obter alívio de um mal-estar psicológico intenso, sendo igualmente referido por meninos e meninas. Razões interpessoais e chamar a atenção de alguém, são menos comumente indicados. Os adolescentes que tem como motivador o desejo de obter alívio desse mal-estar intenso, são mais propen- 
sos a repetir a automutilação, quando comparado com qualquer outro (RASMUSSEN, HAWTON, PHILPOTMORGAN, 2016). Existem duas teorias para as autoagressões, uma descreve uma intensa ansiedade que é crescente até atingir o ponto de se ferir e a outra, é que não ocorreria aumento da ansiedade, mas sim um fato que ligaria essa necessidade de se ferir, como se fosse um interruptor. Em ambos acontece uma dissociação entre $o$ ato de se ferir (provocar dor física) e a busca dos alívios psicológicos (BORONAT et al,2012).

\section{Suicídio}

As associações entre bullying, ideação suicida e suicídio foram bem estabelecidas nas últimas duas décadas (VAN GEEL, VEDDER e TANILON, 2014) ( HOLT, VIVOLO-KANTOR e POLANIN, 2015). Sendo essa associação, mais prevalentes para os alvos e os agentes de bullying, com índices três a cinco vezes maior, quando comparado com os não envolvidos (ESPELAGE e HOLT, 2015).

Um estudo realizado com adolescentes de 10 a 18 anos que frequentavam escolas analisando alunos que já haviam tido uma tentativa de suicido, encontrou que $1,2 \%$ dos adolescentes nunca tinham se evolvido em atos de bullying, $5 \%$ frequentemente agiam como agentes de bullying, 6,5\% foram alvos frequentes de bullying, e 11,1\% alternaram, hora foram alvos hora agentes bullying (BOROWSKY, TALIAFERRO e MC MORRIS,2013). Adolescentes que testemunham o bullying, também correm maior risco. Estudantes que observam o comportamento de bullying são significativamente mais propensos do que aqueles que não estão envolvidos no bullying, a relatar sentimento de tristeza e desamparos, e precisam ser avaliados para sintomas depressivos (RIVERS e NORET, 2013).

Avaliando adolescentes que cometeram suicídio, o envolvimento em atos de bullying foi um dos muitos fatores que precipitaram a morte por suicídio. Um quarto das vítimas de suicídio tiveram problemas escolares, mas apenas $12,4 \%$ desses problemas escolares foram atribuídos ao bullying (KARCH, LOGAN e MCDANIEI, 2013).

Quando se avalia a incidência de suicídio em adolescentes envolvidos em atos de bullying, a depressão e ansiedade aparecem como comorbidades
(Burnstein, Marrocco e KLEINMAN, 2007) (BURNSTEIN, SOURANDER e NIEMELÃ, 2009) (BAUMAN, TOOMEY e WALKER, 2013). Embora a relação entre bullying e depressão já seja bem conhecida e pode ajudar a explicar a relação entre o bullying e o suicídio, o que ainda se pergunta é se os alvos de bullying ficam deprimidos ou se por estarem deprimidos são mais propensos a sofrer bullying? (ESPELAGE e HOLT, 2015). Em pesquisa realizada na Holanda os dados indicaram que ambas suposições são corretas. Os pesquisadores descobriram que alvos de bullying tinham chances significativamente maiores de desenvolver novos problemas psicossomáticos e psicossociais ao longo de um ano letivo em comparação com não alvos. No entanto, os adolescentes com sintomas depressivos preexistentes ou com ansiedade também foram significativamente mais propensos a virem a ser alvos de bullying ao longo do ano letivo (BAUMAN, TOOMEY e WALKER, 2013).

Outros fatores que podem também atuar como comorbidade são: problemas de saúde mental atuais (37\%); namoros abusivos (25\%); história de tentativas de suicídio prévias (18\%); e problemas de abuso de substâncias (16\%) (ESPELAGE e HOLT, 2015). Bem como a relação de suicídio bullying e autoagressão não suicida relatada anteriormente. Adolescentes que praticaram autoagressão não suicida têm risco aumentado para suicídio que pode ser diretamente proporcional à violência do método empregado (OLFSON et al, 2019).

Como possíveis fatores protetores para a ocorrência de suicídio em adolescentes envolvidos em bullying, o bom relacionamento com os pais e amigos e outros adultos, o sentimento que os preocupam com eles, foi o que se mostrou mais significativamente protetores (ESPELAGE e HOLT, 2015).

\section{Conclusão}

A prevalência de bullying vem aumentando em todo mundo, inclusive no Brasil. A infância e adolescência são fases cruciais do desenvolvimento, em particular do cérebro, que faz com que agravos que causem estresses toxico possam levar a mudanças fisiológicas e estruturais.

Essas mudanças acarretarão dificuldades de aprendizagem, de avaliar situações de risco, prejuízo 
da memória de curta e longa duração e ao surgimento de doenças mentais. Não é possível dizer que o bullying possa ser indicado como único fator nos casos de autoagressão não suicida ou no suicídio, mas

\section{REFERÊNCIAS}

ALBUQUERQUE, P. P.; WILLIAMS, L. C. A.; D' AFFONSECA; S. M. Efeitos tardios do bullying e transtorno de estresse pós-traumático: Uma revisão crítica. Psicologia: Teoria e Pesquisa, v. 29, n.1, p. 91-98, Jan-Mar. 2013.

AZEREDO, C. M.; REZENDE, L. F. M.; MALLINSON, P. A. C.; et. al. Progress and setbacks in socioeconomic inequalities in adolescent health-related behaviors in Brazil: results from three cross-sectional surveys 2009-2015. BMJ Open, v. 23, n. 9(3):e025338, Mar, 2019.

BAKKEN, N. W.; GUNTER, W. D. Self-Cutting and Suicidal Ideation among Adolescents: Gender Differences in the Causes and Correlates of Self-Injury. Published online, v. 33, p. 339-356, 06 Mar 2012.

BELLMORE, A. D.; CILLESSEN; A. H. N. Reciprocal influences of victimization, perceived social preference, and self-concept in adolescence. Self-Identity, v. 5, n. 3, p.209-229, July, 2006.

BENEDICT, F. T.; VIVIER, P. M.; GJELSVIK, A. Mental health and bullying in the United States among children aged 6 to 17 years. J Interpers Violence, v. 30 , n. 5, p. 782-95, Mar, 2015.

BENNARDI, M.; MCMAHON, E.; CORCORAN, P.; et. al. Risk of repeated self-harm and associated factors in children, adolescents and young adults. BMC Psychiatry, v. 16, n. 421, 2016.

BORONAT, A.; NOGUEIRA-LIMA, G.; FU, L. L. Autolesão deliberada e suicídio. In Schvartsman BG, Maluf Jr PT, Coordenadores. 1a edição. Psiquiatria da Infância e Adolescência. Barueri: Ed Manole, 2012, p.175-182.

BOROWSKY, I. W.; TALIAFERRO, L. A.; MCMORRIS, B. J. Suicidal thinking and behavior among youth involved in verbal and social bullying: Risk and protective factors. J Adolesc Health, v. 53, p. 4- 12, Jul, 2013. Supl. 1. são fortes as evidencias científicas que associam o bullying a comportamentos relacionados ao suicídio, para isso façamos recomendações baseadas em evidências para melhorar os esforços de prevenção.

BRENDGEN, M.; OUELLET-MORIN, I.; LUPIEN, S.; et. al. Does cortisol moderate the environmental association between peer victimization and depression symptoms? A genetically informed twin study. Psychoneuroendocrinology, v.84, p. 42-50, Oct, 2017.

BRUNSTEIN; K. A, SNIR, A. et al. Association between victimization by bullying and direct self-injurious behavior among adolescence in Europe: a ten-country study. European Child and Adolescent Psychiatry, v. 25, n 11, p. 1183-1193, Mar, 2016.

Buccim, Marques SS, OH D, Harris NB. Toxic stress in children and adolescents. Adv. Pediatrics, v.63, n. 1. P. 403-428, Aug, 2014. - Verificar referencia

CARRION, V. G, WEEMS, C. F.; RICHERT, K.; et al. Decreased prefrontal cortical volume associated with increased, bedtime cortisol in traumatized youth. Biol Psychiatry, v. 1, n. 68(5), p. 491-3, Sep, 2010.

CLAES, L.; LUYCKX, K. I.; BAETENS, Van de Ven M. "Bullying and Victimization, Depressive Mood, and Non-Suicidal Self-Injury in Adolescents: The Moderating Role of Parental Support," Journal of Child and Family Studies, v. 24, n. 11, p. 3363-3371, 2015.

CORNELL, D.; LIMBER, S. P. Law and Policyon the Concept of Bullying at Scholl. American Pychologist, v.70, n.4, p. 333-43, May/June. 2015.

DU, Plessis.; SMEEKENS, S.; CILLESSEN, A. H. N.; et al. Victimization, Cortisol, and Brain Structure. Frontiers in Psychology, v 9, Article 2706, January 2019.

ESBENSEN, F. A.; CARSON, D. C. Consequences of being bullied results from a longitudinal assessment of bullying victimization in a multisite sample of American students. Youth Soc, v. 41, p.209-233, 2009.

ESPELAGE, D. L.; HOLT, M. K. Suicidal ideation and school bullying experiences after controlling for depression and delinquency. J Adolesc Health, v. 53, p. 27-31, 2013. Supl. 1 
FINSTERWALD, C.; ALBERTINI, C. M. Stress and glucocorticoid receptor-dependent mechanisms long-term memory: From adaptive response to psychopathologies. Neurobil Learn Mem, v. 0, p. 17-29, July, 2014.

GARISCH, J. A.; WILSON, M. S. "Prevalence, correlates, and prospective predictors of non-suicidal self-injury among New Zealand adolescents: cross-sectional and longitudinal survey data. Child and Adolescent Psychiatry and Mental Health, v. 9, n. 1, 2015.

HAWTON, K.; HARRISS, L. Deliberate self-harm in young people: characteristics and subsequent mortality in a 20-year cohort of patients presenting to hospital. J Clin Psychiatry, v. 68, n. 10, p. 574, Oct, 2007.

HOLT, M. K.; VIVOLO-KANTOR, A. M.; POLANIN, J. R, et al. Bullying and suicidal ideation and behaviors: a meta-analisis. Pediatrics, v.135 n. 2, p.496509, Feb, 2015.

JUVONEN, J.; WANG, Y.; ESPINOZA, G. Bullying experiences and compromised academic performance across middle school grades. J. Early Adolesc, v 31, n, 1 p.152-173, Fev, 2011.

KARCH, D. L.; LOGAN, J.; McDANIEL, D. D, et al. Precipitating circumstances of suicide among youth aged 10-17 years by sex: Data from the National Violent Death Reporting System, 16 States, 2005- 2008. J Adolesc Health, v. 53, p. 51-3, Jul, 2013. Supl. 1.

KLOMEK, A. B.; KLEINMAN, M.; ALTSCHULER, E.; et al. Suicidal adolescents' experiences with bullying perpetration and victimization during high school as risk factors for later depression and suicidality. J Adolec Health, v. 53, p. 37-42, Jul, 2013. Supl. 1.

KODISH, T.; HERRES, J.; SHEARER, A. et al. Diamond G. Bullying, Depression, and Suicide Risk in a Pediatric Primary Care Sample. Crisi, v.37 n. 3 p. 241-246, may. 2016.

LUPIEN, S. J.; MCEWEN, B. S.; GUNNAR, M. R.; et al. Effects of stress throughout the lifespan on the brain, behaviour and cognition. Nat Rev Neurosci, v. 10, n.6, p. 434-45, Jun, 2009.

MARS, B.; HERON, J.; CRANE, C.; et al. Differences in risk factors for self-harm with and without suicidal intent: findings from the ALSPAC cohort. J Affect Disord, v.168, p. 407-14, Oct, 2014.
MELLO, F. C. M, SILVA, J. C.; OLIVEIRA, W. A.; et al. A prática de bullying entre escolares brasileiros e fatores associados, Pesquisa Nacional de Saúde do Escolar 2015. Ciência e Saúde Coletiva, v. 22, n. 9, p. 2939-2948, 2017.

MC DOUGALL, P.; VAILLANCOURT, T. Long Term Adult Outcomes of Peer Victimization in Childhood and Adolescents. American Psychologist, v.70, n. 4, p. 300-310, may/june, 2015.

MCEWEN, B. S.; GIANAROS, P. J. Stress- and allostasis-induced brain plasticity. Annu Rev Med, v.62, p 431-445, 2011.

MORAN, P.; COFFEY, C.; ROMANIUK, H. et al. The natural history of selfharm from adolescence to young adulthood: a population-based cohort study. Lancet, v. 379, n. 9812, p. 236-43, 2012.

MORGAN, C.; WEBB, R. T.; CARR, M. J. et al. Incidence, clinical management, and mortality risk following self harm among children and adolescents: cohort study in primary care. BMJ, v. 359 j4351, 2017.

NAVEED, S.; WAGAS, A.; AEDMM, K. K.; et al. Association of bullying experiences with depressive symptoms and psychosocial functioning among school going children and adolescents. BMC Res Note, v.2 n.12(1), p. 198, Apr, 2019.

OLFSON, M.; WALL, M.; WANG, S.; et al. Suicide After Deliberate Self-Harm in Adolescents and Young Adults. Pediatrics, v. 141, n.4 p.1-12, April, 2019.

OLWEUS, D. Stability of aggressive reaction patterns in males: A Review. Psychological Bulletin, v. 86, p. $852-875,1979$.

OLWEUS, D. Bullying at school: What we know and what we can do. Blackwell Publishing, v. 140, 1993.

RASMUSSEN, S.; HAWTON, K.; PHILPOTT-MORGAN, S. et al. O'Connor RC. Why Do Adolescents Self-Harm? An Investigation of Motives in a Community Sample. Crisis, v. 37, p 176-183, 2016.

RIVA, P.; ROMERO, L. L. J.; VERGALLITO, A.; et al. Electrified emotions: Modulatory effects of transcranial direct stimulation on negative emotional reactions to social exclusion. Soc Neurosci, v.10, n. 1, p. 46-54, 2015. 
RIVERS, I.; NORET, N. Potential suicide ideation and its association with observing bullying at school. $\mathbf{J}$ Adolesc Health, v. 53, p. 32-6, Jul, 2013. Supl. 1.

RUDOLPH, K. D.; TROOP-GORDON, W.; GRANGER, D. A. Individual differences in biological stress responses moderate the contribution of early peer victimization to subsequent depressive symptoms. Psychopharmacology (Berl), v. 214, n.1, p. 209-19, Mar, 2011.

RUDOLPH, K. D.; MIERNICKI, M. E.; TROOP-GORDON, W.; et al. Adding insult to injury: neural sensitivity to social exclusion is associated with internalizing symptoms in chronically peer-victimized girls. Soc Cogn Affect Neurosc, v.11, n. 5, p.829-842, May, 2016.

SHONKOFF, J. P.; GARNER, A. S. Lifelong Effects of Early Childhood Adversity and Toxic Stress. From The American Academy of Pediatrics. Pediatrics, v. 129, n. 1, p. 232-46, Jan, 2012.

SMITHUIS, L.; KOOL-GOUDZWAARD, N. M. V.; GINKEL, J. M, et al. Self-injurious behaviour in patients with anorexia nervosa: a quantitative study. J Eat Disord, v. 3, n. 6, p. 2-10, Oct, 2018.

TELZER, E. H, MIERNICKI, M. E.; RUDOLPH, K. D. Chronic peer victimization heightens neural sensitivity to risk taking. Dev Psychopathol, v.30, n.1, p. 13-26, Feb, 2018.
UNICEF. An Everyday Lesson End violence in Schools. Disponível em: http://www.unicef.org/publications/files/ Acesso em 03 abr. de 2019.

UNICEF. An unfair start inequality children education. Disponível em: http://www.unicef-irc.org/ publications/pdf/ Acesso em 03 abr. de 2019.

VAN GEEL, M.; VEDDER, P.; TANILON, J. Relationship between peer victimization, cyberbullying, and suicide in children and adolescents: A meta-analysis. JAMA Pediatr, v.168 n. 5 p. 435-442, May, 2014.

VARELA-BESTEIRO, $O$ et al. Suicidal ideation and self-injurious behavior in adolescents with eating disorders. Actas Esp Psiquiatr, v.45, n. 4, p.157-66, Jul, 2017.

WILL, G. J.; VAN LIER, P. A.; CRONE, E. A.; et al. Chronic Childhood Peer Rejection is Associated with Heightened Neural Responses to Social Exclusion During Adolescence. J Abnorm Child Psychol, v.44, n.1, p. 43-55, Jan, 2016.

YSTGAARD, M.; ARENSMAN, E.; HAWTON, K.; et al. Deliberate self-harm in adolescents: comparison between those who receive help following selfharm and those who do not. J Adolesc, v. 32, p.875$91,2008$.

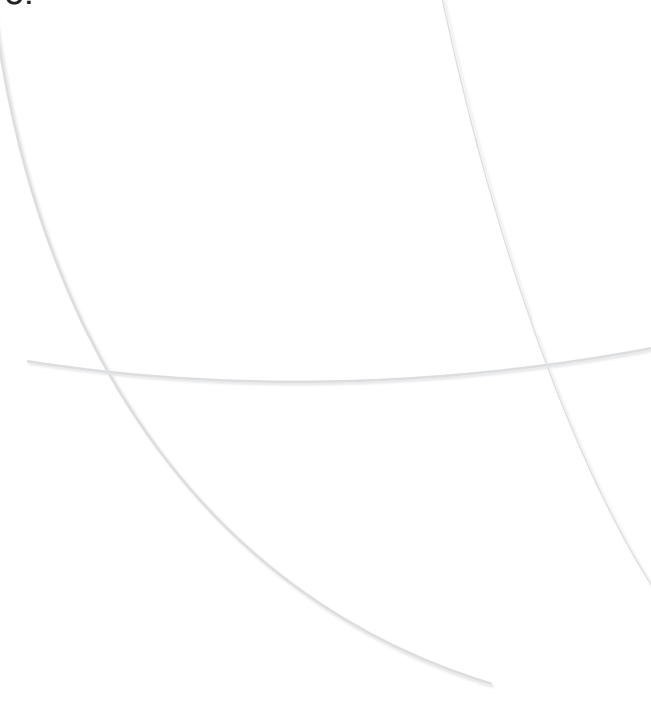

